\title{
Benchmarking several van der Waals dispersion approaches for the description of intermolecular interactions
}

\author{
Julien Claudot, ${ }^{1, \text { a) }}$ Won June Kim, ${ }^{2}$ Anant Dixit, ${ }^{1}$ Hyungjun Kim, ${ }^{3}$ Tim Gould, ${ }^{4}$ Dario \\ Rocca, ${ }^{5}$ and Sébastien Lebègue ${ }^{5, b)}$ \\ 1) Université de Lorraine, CRM², UMR 7036, 54506 Vandœuvre-lès-Nancy, \\ France \\ 2) CNRS, CRM², UMR 7036, 54506 Vandouvre-lès-Nancy, \\ France \\ 3) Department of Chemistry and Graduate School of EEWS, \\ Korea Advanced Institute of Science and Technology, 291 Daehak-ro, Yuseong-gu, \\ Daejeon 34141, Korea \\ 4) Qld Micro- and Nanotechnology Centre, Griffith University, Nathan, Qld 4111, \\ Australia \\ 5) Université de Lorraine and CNRS, CRM², UMR 7036, \\ 54506 Vandœuvre-lès-Nancy, France
}

(Dated: 31 January 2018)

Seven methods, including three van der Waals density functionals (vdW-DFs) and four different variants of the Tkatchenko-Scheffler (TS) methods, are tested on the A24, L7, and Taylor et al.'s "blind" test sets. It is found that for these systems, the vdW-DFs perform better that the TS methods. In particular, the vdW-DF-cx functional gives binding energies that are the closest to the reference values, while the many body correction of TS does not always lead to an improvement in the description of molecular systems. In light of these results, several directions for further improvements to describe van der Waals interactions are discussed.

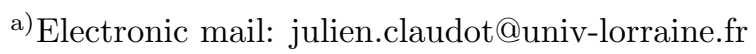

b)Electronic mail: sebastien.lebegue@univ-lorraine.fr
} 


\section{INTRODUCTION}

Over the last two decades, theoretical and computational developments have led to the widespread use of density functional theory $(\mathrm{DFT})^{1}$ in different fields of science. The success of DFT comes from its ability to reformulate the many-body Coulomb problem in the form of an easy to approximate exchange-correlation potential and energy ${ }^{2}$. However, one of the most critical failures of standard exchange-correlation functionals is their inability to describe correctly dispersive forces. Recently, efforts to cure this shortcoming have been made at different levels of theory, ranging from: i) corrections to existing semi-local functionals (the so-called family of DFT $+\mathrm{D}$ methods $)^{3-9}$,ii) functionals taking into account non-locality explicitly ${ }^{10-12}$, iii) methods expressing the exchange-correlation contribution in terms of response functions such as the random phase approximation (RPA) and beyondRPA methods ${ }^{13-17}$, through to iv) advanced wavefunction methods such as coupled cluster and quadratic configuration interaction methods ${ }^{18-23}$. The choice of one method over another is usually dictated by the size of the system - although wavefunction methods are often precise, their computational cost makes them impossible to use for systems of a certain size. On the other hand DFT+D and vdW-DF methods are computationally relatively cheap. But although the accuracy of these methods has been scrutinized in the past ${ }^{5,9,12,24-28}$, it is presently still difficult to have a complete picture of their respective performances. It is thus difficult to make an informed choice about which method to use for a given problem, by trading off speed and accuracy.

In this paper, we provide additional benchmarking on the performance of the Tkatchenko and Scheffler (TS $)^{6}$, non-local van der Waals density functionals (vdW-DFs) and many-body dispersion (MBD) methods on the A24 dataset ${ }^{29}$, the $\mathrm{L} 7^{30}$ dataset, and on a set of dimers recently studied by Taylor et $a .^{31}$. These datasets have been chosen to cover a wide range of chemical situations, from purely dispersive interactions to hydrogen bonds.

The layout of the paper is organized as follows: in the following section, Sec. II, we briefly present the theoretical formalism of the methods used here. Then the interactions energies, calculated for the A24 test set ${ }^{29}$, the L7 test set ${ }^{30}$ and the "blind" set of molecules proposed by Taylor et $a l .{ }^{31}$, are shown and analyzed in Sec. III. Then, figure 4 provides a visual summary of key results. Finally Sec. IV contains our conclusions. 


\section{THEORY AND COMPUTATIONAL DETAILS}

In this section, we summarize the general theoretical background of the methods used in this work, from non-local van der Waals density functionals to the differents schemes proposed by Tkatchenko and Scheffler ${ }^{6}$ (TS) and their variants, which include self-consistent screening $^{32}$ (SCS) or many-body dispersion (MBD) interactions ${ }^{32-34}$. Summaries on some of the theoretical advantages and disadvantages of these approaches can also be found in Refs. ${ }^{35,36}$.

\section{A. Nonlocal van der Waals density functionals (vdW-DFs)}

In 2004, Dion et al. ${ }^{10}$ proposed a functional (named vdW-DF1) that includes vdW forces in a seamless fashion by dividing the exchange-correlation energy functional into three pieces:

$$
E_{\mathrm{xc}}[n]=E_{\mathrm{x}}[n]+E_{\mathrm{c}}^{0}[n]+E_{\mathrm{c}}^{\mathrm{nl}}[n] .
$$

The first term on the right hand side of Eq. 1 is the exchange energy functional usually chosen among GGA exchange functionals, the second term is the local correlation energy functional which is approximated to the LDA correlation functional, and the last term is the non-local correlation functional defined as:

$$
E_{\mathrm{c}}^{\mathrm{nl}}[n]=\frac{1}{2} \iint d^{3} r d^{3} r^{\prime} n(\mathbf{r}) \phi\left(\mathbf{r}, \mathbf{r}^{\prime}\right) n\left(\mathbf{r}^{\prime}\right)
$$

where $n(\mathbf{r})$ is the electron density at the point $\mathbf{r}$ and $\phi\left(\mathbf{r}, \mathbf{r}^{\prime}\right)$ is a kernel designed to capture non-local correlation effects. With the aid of the plasmon-pole approximation for the inverse dielectric function, the non-local kernel is written as:

$$
\phi\left(\mathbf{r}, \mathbf{r}^{\prime}\right)=\frac{2 m e^{4}}{\pi^{2}} \int_{0}^{\infty} a^{2} d a \int_{0}^{\infty} b^{2} d b W(a, b) T\left(\nu(a), \nu(b), \nu^{\prime}(a), \nu^{\prime}(b)\right)
$$

where

$$
\begin{aligned}
W(a, b)= & 2\left[\left(3-a^{2}\right) b \cos b \sin a+\left(3-b^{2}\right) a \cos a \sin b\right. \\
& \left.+\left(a^{2}+b^{2}-3\right) \sin a \sin b-3 a b \cos a \cos b\right] / a^{3} b^{3},
\end{aligned}
$$

and

$$
T(w, x, y, z)=\frac{1}{2}\left[\frac{1}{w+x}+\frac{1}{y+z}\right]\left[\frac{1}{(w+y)(x+z)}+\frac{1}{(w+z)(y+x)}\right]
$$


$\nu$ and $\nu^{\prime}$ in Eq. 3 are given by $\nu(y)=y^{2} / 2\left[1-\exp \left(-4 \pi y^{2} / 9 d^{2}\right)\right]$ and $\nu^{\prime}(y)=y^{2} / 2[1-$ $\left.\exp \left(-4 \pi y^{2} / 9 d^{\prime 2}\right)\right]$ with $d\left(\mathbf{r}, \mathbf{r}^{\prime}\right)=\left|\mathbf{r}-\mathbf{r}^{\prime}\right| q_{0}(\mathbf{r})$ and $d^{\prime}\left(\mathbf{r}, \mathbf{r}^{\prime}\right)=\left|\mathbf{r}-\mathbf{r}^{\prime}\right| q_{0}\left(\mathbf{r}^{\prime}\right)$. Therefore, the non-local correlation energy depends on distances $\left|\mathbf{r}-\mathbf{r}^{\prime}\right|$ via $d\left(\mathbf{r}, \mathbf{r}^{\prime}\right)$ and $d^{\prime}\left(\mathbf{r}, \mathbf{r}^{\prime}\right)$, which are scaled by an effective wavenumber:

$$
q_{0}(\mathbf{r})=-\frac{4 \pi}{3} \epsilon_{\mathrm{xc}}^{\mathrm{LDA}} n(\mathbf{r})-\frac{Z_{\mathrm{ab}}}{9} s^{2}(\mathbf{r}) k_{\mathrm{F}}(\mathbf{r})
$$

with the Fermi wave vector $k_{\mathrm{F}}^{3}(\mathbf{r})=3 \pi^{2} n(\mathbf{r})$ and the reduced gradient $s(\mathbf{r})=\nabla n(\mathbf{r}) / 2 k_{\mathrm{F}}(\mathbf{r}) n(\mathbf{r})$.

The original vdW-DF1 of Dion et al. adopted the revPBE functional ${ }^{10,37,38}$ for the exchange and $Z_{\mathrm{ab}}=-0.8491$ in the non-local correlation functional to reproduce the correlation energy at the slowly varying density. It was found that the vdW-DF1 functional overestimates equilibrium distances due to the too repulsive revPBE exchange functional, and underestimates hydrogen-bond strength ${ }^{39}$.

To solve these problems, a second version, named vdW-DF2, was proposed by Lee $e t a l{ }^{40}$ by replacing the revPBE exchange with the less repulsive PW $86^{41}$ exchange. They have used $Z_{\mathrm{ab}}=-1.887$ to reproduce the asymptotic behavior of the correlation energy at the high density limit, since it provides a more accurate approximation for atoms and molecules than the gradient expansion used in vdW-DF1. Recently, Hamada proposed an improved version of the vdW-DF2 functional, which is called rev-vdW-DF2 (also called revB86bDF2) ${ }^{42}$, by pairing the revised B86b exchange functional (called B86R) with the non-local functional of vdW-DF2. The B86R functional was designed to be as the B86b functional ${ }^{43}$ at the large gradient limit but to behave as the second order of gradient in the low gradient region. At the same time, Berland and Hyldgaard ${ }^{12}$ proposed another exchange functional to be coupled with the non-local functional of vdW-DF1. This new functional, which is called LV-PW86r functional, was designed to switch the gradient enhancement factor from Langreth-Vosko (LV) ${ }^{44}$ at small values of the gradient region to PW86r at large values. This combination led to the creation of the vdW-DF-cx functional, where cx stands for consistent exchange. Several tests have been conducted ${ }^{45}$ and have shown that this functional improves the description of the binding energy of weakly interacting molecular and bulk systems.

Here we tested the performance of the two most recent functionals (rev-vdW-DF2 and vdW-DF-cx) as well as the historical vdW-DF2. 


\section{B. The Tkatchenko-Scheffler method and its variants}

An alternative to dispersion functionals is to apply a semi-empirical dispersion correction. Many variants of corrections exist ${ }^{46-48}$. Here we focus on the Tkatchenko-Scheffler (TS) method, and its descendants.

The dispersion corrected total energy is written $\mathrm{as}^{3}$ :

$$
E_{\mathrm{DFT}-\mathrm{disp}}=E_{\mathrm{KS}-\mathrm{DFT}}+E_{\mathrm{disp}}
$$

where $E_{\mathrm{KS}-\mathrm{DFT}}$ is the DFT energy as obtained, for instance, with PBE. $E_{\text {disp }}$ is, in analogy to the PBE-D2 given by Grimme ${ }^{4}$, the $\mathrm{TS}^{6}$ dispersion energy correction written as:

$$
E_{\text {disp }}=-\frac{1}{2} \sum_{A=1}^{N} \sum_{B=1, B \neq A}^{N} \frac{C_{6 A B}}{R_{A B}^{6}} f_{\text {damp }}\left(R_{A B} ; s_{\mathrm{R}}\right) \text {, }
$$

where $C_{6 A B}$ denotes the dispersion coefficient for an atom pair $A B$ while $R_{A B}^{6}$ is the distance between two atoms. $f_{\text {damp }}$ is a Fermi-type damping function, which decays at small $r$ fast enough to allow the correction to be negligible when the distance between atoms is lower than the typical vdW distance. This function contains a free parameter, denoted $s_{\mathrm{R}}$, which is adjusted according to the exchange-correlation functional used. A value of 0.94 is known to be optimal for the PBE functional ${ }^{49}$.

For a given atom in its environment, the polarizability $\alpha_{A}^{\mathrm{TS}}$ and the dispersion coefficient $C_{6 A A}^{\mathrm{TS}}$ are obtained by rescaling the free atom polarizability $\alpha_{A}^{\text {free }}$ and dispersion coefficient $C_{6 A A}^{\mathrm{free}}$ as:

$$
\alpha_{A}^{\mathrm{TS}}=\frac{V_{A}^{\mathrm{eff}}}{V_{A}^{\text {free }}} \alpha_{A}^{\text {free }}
$$

and

$$
C_{6 A A}^{\mathrm{TS}}=\left(\frac{V_{A}^{\mathrm{eff}}}{V_{A}^{\mathrm{free}}}\right)^{2} C_{6 A A}^{\mathrm{free}} .
$$

The scaling factor $V_{A}^{\text {eff }} / V_{A}^{\text {free }}$ is the ratio between the volume occupied by an atom in his

environment $V^{\text {eff }}$ and the free non-interacting reference $V^{\text {free }}$. The volumes are estimated using a Hirshfeld partitioning of the electron density. Then the dipole-dipole dispersion parameter for an atom-pair $C_{6 A B}$ is computed by following the combination rule:

$$
C_{6 A B}=\frac{2 C_{6 A A} C_{6 B B}}{\left[\frac{\alpha_{B}}{\alpha_{A}} C_{6 A A}+\frac{\alpha_{A}}{\alpha_{B}} C_{6 B B}\right]} .
$$




\section{The self consistent screening scheme}

The TS scheme includes on-site screening but does not include long-range electrostatic screening extending beyond the range of the exponentially decaying densities. This is corrected in the Tkatchenko and Scheffler + self-consistent screening $(\mathrm{TS}+\mathrm{SCS})^{7}$ method, which includes long-range screening behavior through the frequency-dependent screened polarizability $\alpha_{A}^{\mathrm{SCS}}(i \omega)$ found by solving the coupled equations:

$$
\alpha_{A}^{\mathrm{SCS}}(\mathbf{r} ; i \omega)=\alpha_{A}^{\mathrm{TS}}(\mathbf{r} ; i \omega)-\alpha_{A}^{\mathrm{TS}}(\mathbf{r} ; i \omega) \sum_{A \neq B} \tau_{A, B} \alpha_{B}^{\mathrm{SCS}}(\mathbf{r} ; i \omega)
$$

where $\tau_{A, B}$ is the dipole-dipole interaction tensor. Thus, both the short-range (via the TS scheme) and the long-range (via the SCS scheme) electrostatic screenings are contained in the solution of the SCS equation for every frequency of the electric field. The coefficients $C_{6 A A}$ are calculated using the frequency-dependent polarizability and the Casimir-Polder integral $^{50}$

$$
C_{6 A A}=\frac{3}{\pi} \int_{0}^{\infty} \alpha_{A}^{\mathrm{SCS}}(i \omega) \alpha_{A}^{\mathrm{SCS}}(i \omega) d \omega,
$$

which are used in (8) for the energy. As before, for the PBE functional, the optimal value for the adjustable parameter $s_{\mathrm{R}}$ is set equal to 0.97 .

\section{The many body dispersion scheme}

A non-negligible part of the dispersion interaction comes from many-body interactions which are not described completely by the TS and TS+SCS schemes, although they are known to be of substantial importance ${ }^{51}$ in the description of molecular systems. For instance, this type of interaction can be modelled $^{7}$ by a set of quantum harmonic oscillators (QHOs) coupled through a dipole-dipole potential. In particular, it has been shown ${ }^{33}$ that

the interaction energy obtained as the difference between the eigenvalues of the coupled system of QHOs and the eigenvalues of the uncoupled system is equal to the RPA dispersion energy:

$$
E_{\mathrm{disp}}=\int_{0}^{\infty} \frac{d \omega}{2 \pi} \ln \operatorname{det}\left[C^{\mathrm{RPA}}(i \omega)\right],
$$

of the QHOs, in which the $C^{\mathrm{RPA}}$ matrix is defined as:

$$
C^{\mathrm{RPA}}(i \omega)=\delta_{p q}+\left(1-\delta_{p q}\right) \alpha_{p}(i \omega) \tau_{p q}
$$


where $\tau_{p q}$ is the dipole-dipole interaction tensor and $\alpha_{p}(i \omega)$ is an atomic polarizability. In practice $^{33}$, a range separation is introduced in the dipole-dipole interaction so that Eq. (14) is further modified to incorporate both the SCS and MBD approaches, an approach known as the MBD@rsSCS scheme ${ }^{33}$.

\section{The fractional ions scheme}

Further refinements were proposed by Gould et al. ${ }^{34}$ that led to improvements in more difficult nanosystems. Firstly, by using the gas phase polarizabilities of fractional ions ${ }^{52}$, the ionic nature of the system is fully taken into account. Secondly, the eigenvalues of the system are remapped to avoid unphysical "polarization catastrophes", which can lead to failures in SCS and MBD calculations. The remapping involves using the eigenvalues $x_{n}$ of the Hermitian matrix $X(\omega)=-T_{\mathrm{LR}}^{1 / 2} A_{\mathrm{LR}}(\omega) T_{\mathrm{LR}}^{1 / 2}$, where $A_{\mathrm{LR}}(\omega)$ is obtained using short-range screening on the ionic polarizability model. The MBD dispersion energy is then rewritten as ${ }^{53}$ :

$$
E_{\text {disp }}=-\int_{0}^{\infty} \frac{d \omega}{2 \pi} \sum_{n} \ln \left[1+\tilde{x}_{n}(\omega)\right]-\tilde{x}_{n}(\omega)
$$

where

$$
\tilde{x}_{n}=\left\{\begin{array}{ll}
x_{n} & x_{n} \geq 0 \\
-\left[\operatorname{erf}\left(\frac{\sqrt{\pi}}{2}\left|x_{n}\right|\right)^{4}\right]^{1 / 4} & x_{n}<0
\end{array} .\right.
$$

Unlike the raw eigenvalues $x_{n}(\omega)$, which can unphysically become less than -1 causing the polarization catastrophe, the remapping ensures that $\tilde{x}_{n}(\omega)>-1$.

\section{Computational details}

The calculations presented in this work have been performed using the VASP (Vienna $a b$ initio simulation package $)^{54,55}$ code. To ensure a high level of precision in our calculations, we have used a large cut-off of $1000 \mathrm{eV}$. At the same time, the use of a plane-wave basis set implies the lack of basis set superposition error. Also, sufficiently large cells were used to avoid spurious interactions between periodically repeated images, which were single $\Gamma$ point calculations. In the calculations using the TS method and its variants, the exchangecorrelation energy was obtained using Perdew-Burke-Ernzerhof $(\mathrm{PBE})^{37}$ functional. 
Calculations using the non-local vdW functionals, vdW-DF2 and rev-vdW-DF2 calculations were performed using the implementation ${ }^{25,40,56,57}$ in VASP. VASP was also used for the vdW-DF-cx functional with the implementation of Björkman ${ }^{58}$. Detailed discussion of the MBD implementation in the VASP code can be found in previous publications ${ }^{34,59,60}$ and will not be repeated here.

\section{RESULTS}

\section{A. A24 dataset}

In this section we present our results for the A24 test set proposed by Řezáč and Hobza ${ }^{29}$, which contains 24 molecular dimers of small sizes. This test set composed of small and diverse complexes, covers different types of noncovalent interacting systems, and is ideal to perform high level benchmarking calculation. All dimers were placed in a $17.5 \times 17.5 \times 17.5 \AA^{3}$ box and our results are compared with respect to CCSDT(Q) extrapolated to the complete basis set limit (CBS) values from Řezáč and Hobza ${ }^{29}$.

As indicated in Table I, the mean absolute errors (MAE) with respect to the reference values from Řezáč and Hobza ${ }^{29}$ are respectively $0.143 \mathrm{kcal} / \mathrm{mol}$ for vdW-DF2, 0.133 $\mathrm{kcal} / \mathrm{mol}$ for rev-vdW-DF2 and $0.137 \mathrm{kcal} / \mathrm{mol}$ for vdW-DF-cx. Those errors increase to 0.351, 0.290, 0.309 and $0.294 \mathrm{kcal} / \mathrm{mol}$, respectively for the TS, TS+SCS, MBD@rsSCS and MBD@rsSCS/FI methods. It appears, by comparing to the MAE value of PBE calculations which is equal to $0.404 \mathrm{kcal} / \mathrm{mol}$, that the correction brought by the TS scheme is limited, reducing the error by only $13.1 \%$. This correction increases to $28.2 \%$ with the TS+SCS scheme, to $23.5 \%$ with the MBD@rsSCS method, and to $27.2 \%$ with MBD@rsSCS/FI. On the contrary, the non-local vdW based methods show a reduction of $64.5 \%$ to $67 \%$ of the MAE in comparison with that of the PBE.

Considering the TS, TS+SCS, MBD@rsSCS and MBS@rsSCS/FI schemes, the mean absolute percentage errors (MAPE) shows a slight different behavior from the MAE. Indeed, with respect to $\operatorname{CCSDT}(\mathrm{Q}) / \mathrm{CBS}_{\text {values }}{ }^{29}$, by comparing MAPE equal to $21.96 \%$ and $16.96 \%$, respectively for the TS+SCS and MBD@rsSCS/FI, one can note a decreasing of $5 \%$ while their MAE are relatively similar (within $0.004 \mathrm{kcal} / \mathrm{mol}$ ).

Figure 1 shows the differences between the interaction energies calculated with the dif- 
TABLE I: Interaction energies (in $\mathrm{kcal} / \mathrm{mol}$ ) for the A24 test set obtained with PBE, vdW-DF2, rev-vdW-DF2, vdW-DF-cx, TS, TS+SCS, MBD@rsSCS and MBD@rsSCS/FI methods compared with $\operatorname{CCSDT}(\mathrm{Q}) / \mathrm{CBS}$. The mean absolute errors (MAE), mean errors (ME), the maximum deviations (|MAX|) and the mean absolute percentage errors (MAPE) are provided with respect to the CCSDT(Q)/CBS values $^{29}$.

\begin{tabular}{|c|c|c|c|c|c|c|c|c|c|c|}
\hline \multicolumn{2}{|l|}{ System } & \multirow{2}{*}{\begin{tabular}{|c|}
$\operatorname{CCSDT}(\mathrm{Q}) / \mathrm{CBS}^{29}$ \\
-6.492
\end{tabular}} & \multirow{2}{*}{\begin{tabular}{|c|}
$\mathrm{PBE}$ \\
-7.008
\end{tabular}} & \multicolumn{3}{|c|}{ vdW-DF2 rev-vdW-DF2 vdW-DF-cx } & \multicolumn{4}{|c|}{ PBE-TS PBE-TS+SCS MBD@rsSCS MBD@rsSCS/FI } \\
\hline 1 water... ammonia & $\mathrm{C}_{\mathrm{s}}$ & & & -6.605 & -6.897 & -6.540 & -7.417 & -7.368 & -7.525 & -7.482 \\
\hline 2 water dimer & $\mathrm{C}_{\mathrm{s}}$ & -4.994 & -5.124 & -5.070 & -5.094 & -4.701 & -5.427 & -5.381 & -5.540 & -5.506 \\
\hline $3 \mathrm{HCN}$ dimer & $\mathrm{C}_{\mathrm{s}}$ & -4.738 & -4.730 & -4.930 & -4.797 & -4.540 & -5.055 & -5.036 & -5.133 & -5.141 \\
\hline 4 HF dimer & $\mathrm{C}_{\mathrm{s}}$ & -4.564 & -4.859 & -4.874 & -4.726 & -4.248 & -4.980 & -4.956 & -5.101 & -4.975 \\
\hline 5 ammonia dimer & $\mathrm{C}_{2 \mathrm{~h}}$ & -3.141 & -2.850 & -3.073 & -2.964 & -2.676 & -3.280 & -3.195 & -3.407 & -3.375 \\
\hline 6 methane... HF & $\mathrm{C}_{3 \mathrm{v}}$ & -1.660 & -1.728 & -1.675 & -1.670 & -1.462 & -2.100 & -2.052 & -2.258 & -2.201 \\
\hline 7 ammonia... methane & $\mathrm{C}_{3 \mathrm{v}}$ & -0.771 & -0.705 & -0.874 & -0.723 & -0.654 & -1.023 & -0.999 & -1.002 & -0.999 \\
\hline 8 methane... water & $\mathrm{C}_{\mathrm{s}}$ & -0.656 & -0.545 & -0.767 & -0.593 & -0.571 & -0.800 & -0.788 & -0.810 & -0.806 \\
\hline 9 formaldehyde dimer & $\mathrm{C}_{\mathrm{s}}$ & -4.479 & -4.077 & -4.976 & -4.926 & -4.514 & -4.886 & -4.808 & -5.099 & -5.128 \\
\hline 10 ethene... water & $\mathrm{C}_{\mathrm{s}}$ & -2.564 & -2.463 & -2.744 & -2.700 & -2.564 & -3.165 & -3.094 & -3.200 & -3.168 \\
\hline 11 ethene. . formaldehyde & $\mathrm{C}_{\mathrm{s}}$ & -1.623 & -0.863 & -1.687 & -1.398 & -1.310 & -1.899 & -1.789 & -1.728 & -1.732 \\
\hline 12 ethyne dimer & $\mathrm{C}_{2 \mathrm{v}}$ & -1.529 & -1.233 & -1.609 & -1.463 & -1.455 & -1.776 & -1.786 & -1.860 & -1.852 \\
\hline 13 ethene... ammonia & $\mathrm{C}_{\mathrm{s}}$ & -1.382 & -1.086 & -1.549 & -1.380 & -1.358 & -1.778 & -1.705 & -1.725 & -1.702 \\
\hline 14 ethene dimer & $\mathrm{C}_{2 \mathrm{v}}$ & -1.106 & -0.409 & -1.352 & -1.036 & -1.044 & -1.569 & -1.512 & -1.391 & -1.395 \\
\hline 15 methane... ethene & $\mathrm{C}_{\mathrm{s}}$ & -0.509 & -0.265 & -0.650 & -0.489 & -0.605 & -0.781 & -0.755 & -0.690 & -0.691 \\
\hline 16 borane... methane & $\mathrm{C}_{\mathrm{s}}$ & -1.513 & -1.199 & -1.372 & -1.509 & -1.335 & -2.136 & -2.009 & -2.160 & -2.182 \\
\hline 17 methane... ethane & $\mathrm{C}_{\mathrm{s}}$ & -0.836 & -0.048 & -1.029 & -0.682 & -0.780 & -1.278 & -1.139 & -0.964 & -0.942 \\
\hline 18 methane... ethane & $\mathrm{C}_{3 \mathrm{v}}$ & -0.614 & -0.035 & -0.765 & -0.479 & -0.603 & -0.944 & -0.807 & -0.716 & -0.695 \\
\hline 19 methane dimer & $\mathrm{D}_{3 \mathrm{~d}}$ & -0.539 & -0.039 & -0.706 & -0.430 & -0.559 & -0.839 & -0.723 & -0.627 & -0.601 \\
\hline 20 methane... Ar & $\mathrm{C}_{3 \mathrm{v}}$ & -0.405 & -0.088 & -0.565 & -0.342 & -0.475 & -0.548 & -0.490 & -0.430 & -0.431 \\
\hline 21 ethene... Ar & $\mathrm{C}_{2 \mathrm{v}}$ & -0.365 & -0.098 & -0.541 & -0.354 & -0.525 & -0.503 & -0.497 & -0.434 & -0.447 \\
\hline 22 ethene... ethyne & $\mathrm{C}_{2 \mathrm{v}}$ & +0.794 & +1.657 & +0.773 & +1.021 & +0.943 & +0.541 & +0.595 & +0.751 & +0.749 \\
\hline 23 ethene dimer & $\mathrm{D}_{2 \mathrm{~h}}$ & +0.909 & +1.908 & +0.932 & +1.156 & +1.004 & +0.611 & +0.722 & +0.884 & +0.873 \\
\hline 24 ethyne dimer & $\mathrm{D}_{2 \mathrm{~h}}$ & +1.084 & +1.856 & +1.006 & +1.325 & +1.308 & +0.899 & +0.901 & +1.051 & +1.055 \\
\hline \multicolumn{11}{|c|}{ Total } \\
\hline MAE & & & 0.404 & 0.143 & 0.133 & 0.137 & 0.351 & 0.290 & 0.309 & 0.294 \\
\hline $\mathrm{ME}$ & & & -0.320 & -0.124 & 0.023 & 0.102 & -0.351 & -0.290 & -0.309 & -0.295 \\
\hline$|\mathrm{MAX}|$ & & & 0.999 & 0.497 & 0.447 & 0.465 & 0.925 & 0.866 & 1.033 & 0.990 \\
\hline MAPE (\%) & & & 42.17 & 13.35 & 9.92 & 10.82 & 27.94 & 21.96 & 17.63 & 16.96 \\
\hline
\end{tabular}

ferents functionals and the CCSDT(Q)/CBS values. As can be seen, the differences are contained within an interval of -0.7 to $+0.5 \mathrm{kcal} / \mathrm{mol}$, except for the water-ammonia dimer, for which the TS based methods give differences close to $-1 \mathrm{kcal} / \mathrm{mol}$. Moreover this figure shows the better performance of the non-local vdW methods over the various TS and MBD methods for the H-bonded and mixed-type systems: indeed a systematic overestima- 


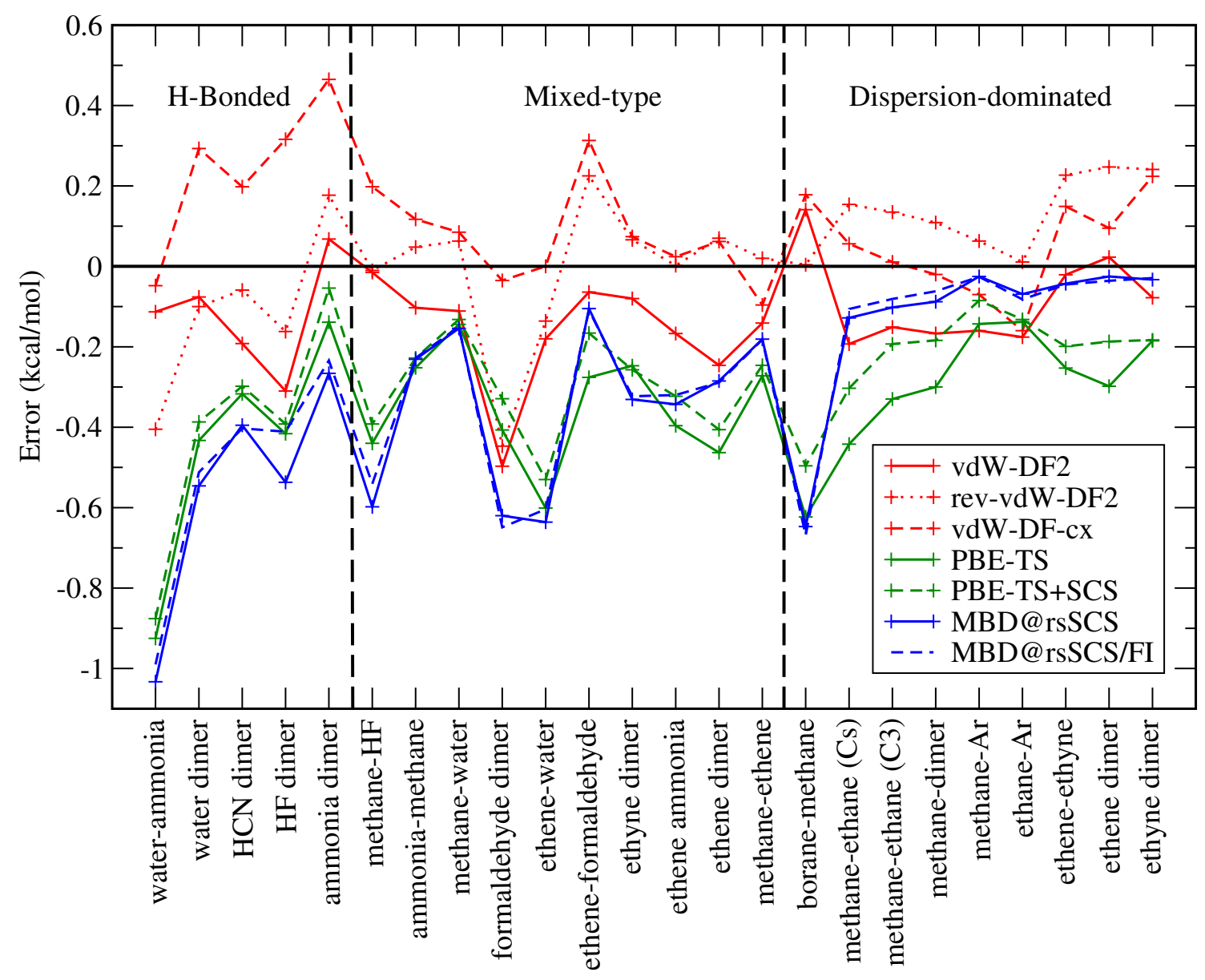

FIG. 1: Differences in kcal/mol for the A24 test for the non-local van der Waals functionals, TS based and many body methods computed with respect to the CCSDT(Q)/CBS reference values ${ }^{29}$.

tion of the binding energies can be found with the TS and MBD methods, whereas a more even distribution of errors is obtained with the vdW-DF flavours. However, except for the borane-methane dimer, the MBD schemes give smaller errors (lower than $-0.1 \mathrm{kcal} / \mathrm{mol}$ ) when applied to dispersion dominated systems.

\section{B. "Blind" test dataset}

To further benchmark the methods of interest here on a different dataset, we have chosen the one created by Taylor et al. ${ }^{31}$, composed of 10 dimers with a range of sizes (from 6 to 32 atoms). In their work, the authors did not release the reference values before the various calculations were conducted, which therefore constituted a "blind" test. In this set, though hydrogen-bonded and dispersion dominated systems still prevail, the dimers 
cannot be strictly classified into either category. Moreover, a majority of dimers of this set are not found in other sets. The geometries used here correspond to the near equilibrium positions, and were taken from the supplementary material of Taylor et al. ${ }^{31}$ optimized at the MP2/aug-cc-pVTZ level of theory. The reference values for the binding energies have been obtained with CCSD(T) extrapolated to CBS by the same authors ${ }^{31}$.

As reported in Table II, the MAE is equal to $3.001 \mathrm{kcal} / \mathrm{mol}$ for $\mathrm{PBE}^{37}$ and shows clearly that in order to describe correctly dispersion dominated system, one has to use semi-empirical correction or non-local functionals to improve the results. Comparing to PBE, the MAE for the whole test set is reduced by $73.5 \%$ and $78.2 \%$ for TS and MBD based schemes respectively, and this reduction increases to $82-83 \%$ for the non-local vdWDF family. Furthermore, the MAPE supports the good performance of the vdW-DF family of methods, especially the vdW-DF2 functional which outperforms all the other methods with a MAPE of $6.74 \%$ to be compared for instance to the value of $10.08 \%$ for vdW-DF-cx, the closest one.

Additionally since Taylor et al. ${ }^{31}$ computed the interaction energies for this test set with the vdW-DF2 functional, we compare our vdW-DF2 results with their results. Our calculations were done using the VASP code with PAW potentials ${ }^{61,62}$ while those from Taylor et al. have been computed with the QBOX code $^{63}$ with norm-conserving pseudopotentials. This may be the reason behind the slight disagreement between the MAE of $0.394 \mathrm{kcal} / \mathrm{mol}$ for the vdW-DF2 results of Ref. 31 and $0.513 \mathrm{kcal} / \mathrm{mol}$ for our vdW-DF2 calculations. By examining more closely the results, most of the difference is due to the nitromethane, nitrobenzene, and EDNA dimers, with deviations of $0.378,0.484$ and $0.535 \mathrm{kcal} / \mathrm{mol}$ respectively between the two vdW-DF2 calculations.

It is important to note here that we obtain positive mean errors for all methods, which indicates a general tendency to underestimate the absolute binding energies of the different systems. This is in contrast to the results for the A24 test set which show the opposite behavior.

\section{L7 dataset}

We then applied the different functionals to the L7 data test set proposed by Sedlak et $a l .{ }^{30}$, which consists of 7 non covalent complexes: "CBH", the octadecane dimer in stacked 
TABLE II: Interaction energies $(\mathrm{kcal} / \mathrm{mol})$ for the molecular dimers from the "blind" test set of Taylor et $a l .31$ obtained with PBE, vdW-DF2, rev-vdW-DF2, vdW-DF-cx, TS, TS+SCS, MBD@rsSCS and MBD@rsSCS/FI methods compared with CCSD(T)/CBS. The mean absolute errors (MAE), mean errors (ME), the maximum deviation (|MAX $\mid$ ) and the mean absolute percentage errors (MAPE) are provided with respect to $\operatorname{CCSD}(\mathrm{T}) / \mathrm{CBS}$ values ${ }^{31}$.

\begin{tabular}{|c|c|c|c|c|c|c|c|c|c|c|}
\hline \multirow{2}{*}{$\frac{\text { System }}{\text { Benzene-methane }}$} & \multicolumn{2}{|c|}{$\operatorname{CCSD}(\mathrm{T}) / \mathrm{CBS}^{31}$ vdW-DF2 $2^{31}$} & \multirow{2}{*}{\begin{tabular}{c|} 
PBE \\
-0.189
\end{tabular}} & \multicolumn{3}{|c|}{ vdW-DF2 rev-vdW-DF2 vdW-DF-cx } & \multicolumn{4}{|c|}{ PBE-TS PBE-TS+SCS MBD@rsSCS MBD@rsSCS/FI } \\
\hline & -1.476 & -1.492 & & -1.460 & -1.264 & -1.417 & -1.705 & -1.686 & -1.441 & -1.459 \\
\hline Ethanol dimer & -2.870 & -2.981 & -0.814 & -2.881 & -2.452 & -2.242 & -3.143 & -3.094 & -2.893 & -2.901 \\
\hline Benzene-water & -2.870 & -2.924 & -1.880 & -2.854 & -2.673 & -2.752 & -3.448 & -3.290 & -3.030 & -2.950 \\
\hline Methylformate dimer & -3.485 & -3.246 & -1.799 & -3.198 & -2.774 & -2.614 & -3.339 & -3.218 & -3.192 & -3.194 \\
\hline Water dimer & -5.049 & -5.031 & -5.160 & -5.070 & -5.124 & -4.727 & -5.460 & -5.414 & -5.574 & -5.541 \\
\hline Nitromethane dimer & -6.393 & -5.972 & -2.570 & -5.594 & -5.242 & -5.053 & -4.647 & -4.369 & -4.737 & -4.765 \\
\hline Nitrobenzene dimer & -7.110 & -5.785 & -2.705 & -5.301 & -6.091 & -6.826 & -6.586 & -5.723 & -4.763 & -4.965 \\
\hline Imidazole dimer & -10.207 & -9.501 & -9.458 & -9.503 & -10.135 & -10.165 & -10.658 & -10.589 & -10.741 & -10.772 \\
\hline EDNA dimer & -12.360 & -11.441 & -4.628 & -10.906 & -11.095 & -10.905 & -10.452 & -9.791 & -10.451 & -10.545 \\
\hline FOX-7 dimer & -12.700 & -12.569 & -10.942 & -12.716 & -12.630 & -12.402 & -12.969 & -12.767 & -13.181 & -13.135 \\
\hline \multicolumn{11}{|c|}{ Total } \\
\hline MAE & & 0.394 & 3.001 & 0.513 & 0.519 & 0.542 & 0.654 & 0.791 & 0.796 & 0.720 \\
\hline ME & & 0.358 & 2.978 & 0.503 & 0.503 & 0.542 & 0.211 & 0.459 & 0.452 & 0.429 \\
\hline$|\mathrm{MAX}|$ & & 1.325 & 9.815 & 1.809 & 1.265 & 1.455 & 1.908 & 2.569 & 2.347 & 2.145 \\
\hline MAPE (\%) & & 5.47 & 52.45 & 6.74 & 10.15 & 10.08 & 11.42 & 12.77 & 11.10 & 10.25 \\
\hline
\end{tabular}

parallel conformation is representative of aliphatic dispersion interaction, "GGG", a stacked guanine trimer arranged as in DNA is representative of the aromatic stacking $\pi \ldots \pi$ dispersion interaction, "PHE", a trimer of phenylalanine residues in mixed H-bonded-stacked conformation is representative of a mixed interaction between dispersion and H-bonds, "C3A" is a stacked circumcoronene... adenine dimer, "C3GC" is a stacked circumcoronene and Watson-Crick hydrogen-bonded guanine-cytosine dimer, "C2C2PD" is a parallel displaced stacked coronene dimer, and "GCGC" is a stacked Watson-Crick hydrogen bonded guaninecytosine dimers arranged as in DNA, the last four being representative of strong aromatic dispersion interaction. Their sizes range from 48 to 112 atoms, which allows to test the behaviour of the methods of interest for relatively large systems. In the original publication ${ }^{30}$, the geometries for six of the complexes, CBH, C3A, GCGC, C3GC, GGG and PHE were determined at the DFT-D TPSS/TZVP ${ }^{64}$ level with no constrains, while the C2C2PD geometry was optimized at the QCISD(T) level of theory. The binding energies were obtained ${ }^{30}$ at the QCISD $(\mathrm{T}) / \mathrm{CBS}$ level of theory.

As seen in Table III, the different MAE with respect to the reference are largely superior 


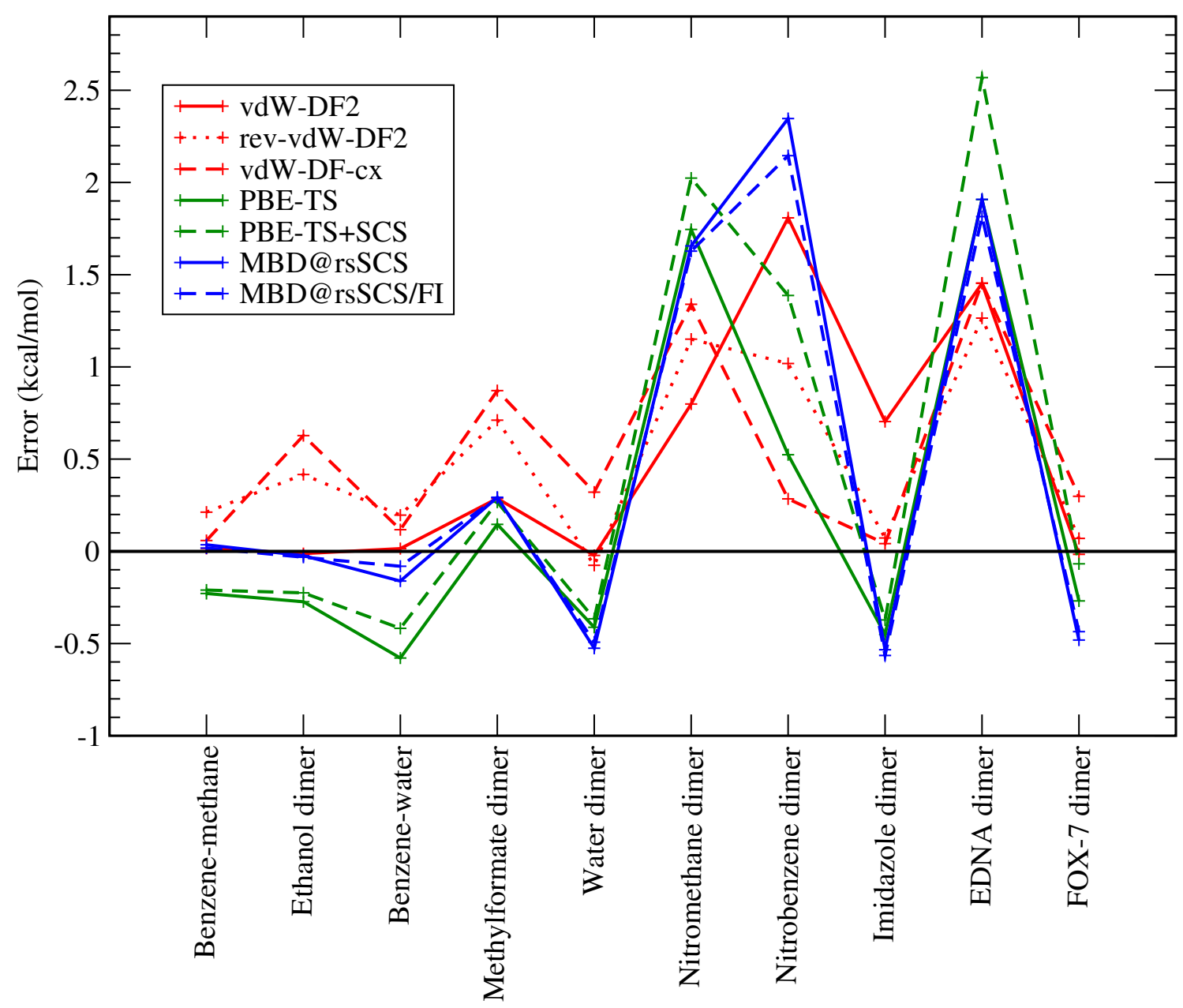

FIG. 2: Differences in $\mathrm{kcal} / \mathrm{mol}$ for the "blind" test set of Taylor et al. ${ }^{31}$ for the non-local van der Waals functionals, TS based and many body methods computed with respect to $\operatorname{CSSD}(\mathrm{T}) / \mathrm{CBS}$ reference values.

to the chemical accuracy of $1 \mathrm{kcal} / \mathrm{mol}$. The difference are especially large for the MBD methods where the MAE is equal to $5.937 \mathrm{kcal} / \mathrm{mol}$ for the MBD with range-separation self consistent screening and equal to $5.790 \mathrm{kcal} / \mathrm{mol}$ for the same scheme with fractional ions. Except for the PBE results, shown here to emphasize the improvement brought on by the different methods, the maximum deviations are found with the MBD methods, with particularly large errors for the $\mathrm{C} 2 \mathrm{C} 2 \mathrm{PD}$ and $\mathrm{C} 3 \mathrm{GC}$ complexes which is reflected by the MAPE, which are respectively 31.27\% and 30.94\% for MBD@rsSCS and MBD@rsSCS/FI. We also observe that the vdW-DF family of methods does not always give the best results: indeed, the TS scheme shows a slightly lower MAE value than vdW-DF2; 2.919 and 3.106 $\mathrm{kcal} / \mathrm{mol}$ respectively.

As seen before for the data set of Taylor et al., the positive mean error demonstrates a 
TABLE III: Interaction energies $(\mathrm{kcal} / \mathrm{mol})$ for the test set L7 of Sedlak et al. ${ }^{30}$ obtained with PBE, vdW-DF2, rev-vdW-DF2, vdW-DF-cx, TS, TS+SCS, MBD@rsSCS and MBD@rsSCS/FI methods compared with QCISD(T)/CBS. The mean absolute errors (MAE), mean errors (ME), the maximum deviation $(|\mathrm{MAX}|)$ and the mean absolute percentage errors (MAPE) are provided with respect to QCISD $(\mathrm{T}) / \mathrm{CBS}_{\text {values }}{ }^{30}$.

\begin{tabular}{|c|c|c|c|c|c|c|c|c|c|}
\hline \multirow{2}{*}{$\frac{\text { System }}{1 \mathrm{C} 2 \mathrm{C} 2 \mathrm{PD}}$} & \multirow{2}{*}{\begin{tabular}{|c|}
$\mathrm{QCISD}(\mathrm{T}) / \mathrm{CBS}^{30}$ \\
-24.36
\end{tabular}} & \multirow{2}{*}{\begin{tabular}{|c|} 
PBE \\
+5.410
\end{tabular}} & \multicolumn{3}{|c|}{ vdW-DF2 rev-vdW-DF2 vdW-DF-cx } & \multicolumn{4}{|c|}{ PBE-TS PBE-TS+SCS MBD@rsSCS MBD@rsSCS/FI } \\
\hline & & & -18.542 & -19.345 & -21.487 & -20.465 & -18.379 & -13.714 & -14.505 \\
\hline $2 \mathrm{C} 3 \mathrm{~A}$ & -18.19 & +2.147 & -14.871 & -15.537 & -17.642 & -15.618 & -14.603 & -11.070 & -11.375 \\
\hline 3 C3GC & -31.25 & +5.157 & -25.627 & -26.816 & -29.946 & -26.271 & -25.305 & -18.898 & -19.278 \\
\hline $4 \mathrm{CBH}$ & -11.06 & -0.469 & -16.006 & -12.768 & -14.757 & -13.497 & -15.324 & -11.458 & -11.746 \\
\hline 5 GCGC & -14.37 & +3.869 & -13.754 & -13.452 & -15.288 & -12.846 & -12.410 & -9.579 & -9.426 \\
\hline 6 GGG & -2.40 & +3.900 & -2.625 & -2.203 & -3.264 & -2.889 & -2.813 & -1.466 & -1.461 \\
\hline $7 \mathrm{PHE}$ & -25.76 & -17.250 & -24.568 & -24.557 & -24.911 & -21.224 & -21.399 & -20.439 & -20.444 \\
\hline \multicolumn{10}{|c|}{ Total } \\
\hline MAE & & 18.632 & 3.106 & 2.304 & 1.579 & 2.919 & 3.787 & 5.937 & 5.790 \\
\hline ME & & 18.632 & 1.628 & 1.816 & 0.014 & 2.083 & 2.451 & 5.824 & 5.594 \\
\hline$|\mathrm{MAX}|$ & & 36.407 & 5.918 & 5.015 & 3.697 & 4.979 & 5.981 & 12.352 & 11.972 \\
\hline MAPE $(\%)$ & & 119.93 & 17.59 & 12.01 & 14.01 & 16.67 & 21.37 & 31.27 & 30.94 \\
\hline
\end{tabular}

tendency to underestimate the binding energy, even for larger systems. Only the vdW-DFcx seems to find a balance between overestimating and underestimating the binding energy for the different systems, with a mean error of $0.014 \mathrm{kcal} / \mathrm{mol}$ and a MAE of $1.579 \mathrm{kcal} / \mathrm{mol}$ with respect to $\mathrm{QCISD}(\mathrm{T}) / \mathrm{CBS}$ values.

\section{DISCUSSION AND CONCLUSIONS}

In this work, we tested seven different methods, from semi-empirical corrections to nonlocal van der Waals functionals and many-body dispersion methods, in order to compare their ability to describe intermolecular interactions, which are of major importance in understanding and describing molecular complexes. When compared to CCSD $(\mathrm{T})$ results at the complete basis set limit and its derivative, which are considered as the "Gold Standard" of computational chemistry ${ }^{29}$ but come with a correspondingly high computational cost, we have found the following results:

- Concerning the A24 test set, all the methods give results that are within the chemical accuracy of $1 \mathrm{kcal} / \mathrm{mol}$. When compared with $\operatorname{CCSDT}(\mathrm{Q}) / \mathrm{CBS}$ reference values, the 


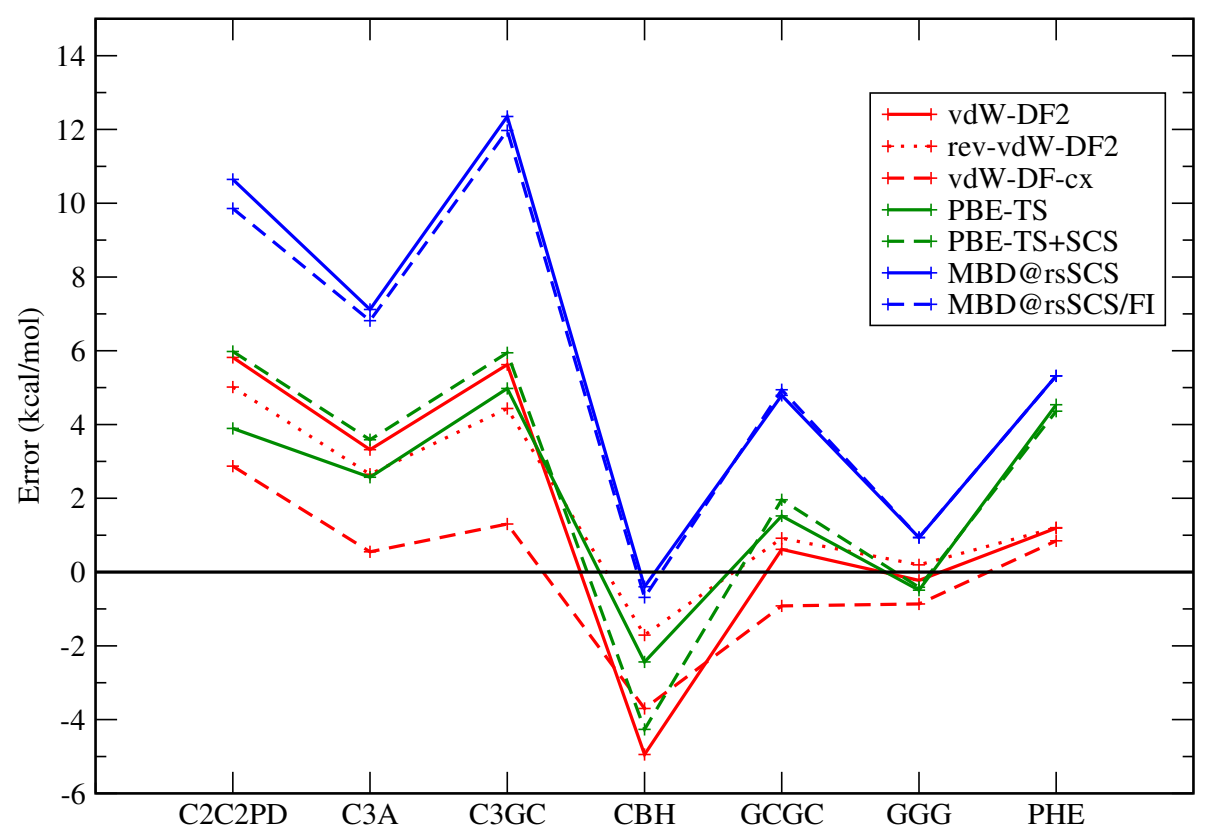

FIG. 3: Differences in kcal/mol for the test set L7 from Sedlak et al. ${ }^{30}$ for the non-local van der Waals functionals, TS based and many body methods computed with respect to QCISD $(\mathrm{T}) / \mathrm{CBS}$ reference values. $^{30}$

non-local vdW functionals have a MAE about 50\% lower than the TS based and MBD methods.

- Almost the same trend is observed among the methods for the "blind" test set of Taylor et al. - the MAE for the vdW-DF methods show a better accuracy of approximately $0.2 \mathrm{kcal} / \mathrm{mol}$ compared to the TS and MBD schemes.

- In the case of the L7 test set, composed by relatively large systems up to 112 atoms, chemical accuracy is not reached and some mean absolute errors or maximum deviations values shows in particular with the TS and MBD methods. We again observe that the non-local vdW-DF family provides the best results, specifically the vdW-DFcx method. The relatively poor performance (compared to the other test sets) of the different schemes is not totally surprising since it is expected that absolute errors tend to increase with the size of the systems.

We summarize all our results in Table IV, Table V and Figure 4: overall, the vdw-DF functionals perform better than the TS and MBD methods. In particular, the vdW-DF-cx functional is the method that performs the best among all the methods that we have tested. 
TABLE IV: Mean Absolute Error (MAE) (kcal/mol) of interaction energies for the three differents test sets are reminded and the MAE obtained for the totality of molecular pairs (41 molecules) composing those test sets with PBE, vdW-DF2, rev-vdW-DF2, vdW-DF-cx, TS, TS+SCS, MBD@rsSCS and MBD@rsSCS/FI.

\begin{tabular}{|c|c|c|c|c|c|c|c|c|}
\hline & \multicolumn{8}{|c|}{ PBE vdW-DF2 rev-vdW-DF2 vdW-DF-cx PBE-TS PBE-TS+SCS MBD@rsSCS MBD@rsSCS/FI } \\
\hline A24 test set & 0.404 & 0.143 & 0.133 & 0.137 & 0.351 & 0.290 & 0.309 & 0.295 \\
\hline "Blind" test set & 3.001 & 0.513 & 0.519 & 0.542 & 0.654 & 0.790 & 0.796 & 0.750 \\
\hline L7 test set & 18.632 & 3.106 & 2.304 & 1.579 & 2.919 & 3.787 & 5.937 & 5.790 \\
\hline Total & 4.149 & 0.739 & 0.598 & 0.482 & 0.863 & 1.009 & 1.389 & 1.344 \\
\hline
\end{tabular}

TABLE V: Mean Absolute Percentage Error (MAPE) (\%) of interaction energies for the three differents test sets are reminded and the MAPE obtained for the totality of molecular pairs (41 molecules) composing those test sets with PBE, vdW-DF2, rev-vdW-DF2, vdW-DF-cx, TS, TS+SCS, MBD@rsSCS and MBD@rsSCS/FI.

\begin{tabular}{|c|c|c|c|c|c|c|c|c|}
\hline & PBE & dW-DF & $-v d W-D$ & W-DF- & BE-T & E-TS+ & 3D@rsS & @rsSCS/FI \\
\hline A24 test set & 42.17 & 13.35 & 9.92 & 10.82 & 27.94 & 21.96 & 17.63 & 16.96 \\
\hline "Blind" test set & 52.45 & 6.74 & 10.15 & 10.08 & 11.42 & 12.77 & 11.10 & 10.25 \\
\hline L7 test set & 119.93 & 17.59 & 12.01 & 14.01 & 16.67 & 21.37 & 31.27 & 30.94 \\
\hline Total & 57.95 & 12.46 & 10.33 & 11.18 & 21.99 & 19.62 & 18.37 & 17.71 \\
\hline
\end{tabular}

Surprisingly, the TS method without further corrections gives MAE results that are closer to the reference values than more advanced schemes such the TS-SCS, the MBD@rsSCS, and the the MBD@rsSCS/FI that include more complete treatment of non-additivity ${ }^{35}$. The MAE is dominated by the larger errors in the larger molecules, however, and may thus reflect a better performance of TS in some of the largest systems. Indeed, the picture changes when we consider the MAPE, where the two MBD-based methods outperform the two TS methods.

One other point is worth mentioning here. Our tests were all evaluated using geometries that had been optimized using higher level theories. Thus our calculations cannot test the ability of the dispersion corrections to calculate forces, which are important for calculating structures and carrying out molecular dynamics. For example, it has been shown that some of the vdW-DF functionals overestimate lattice parameters significantly in layered 


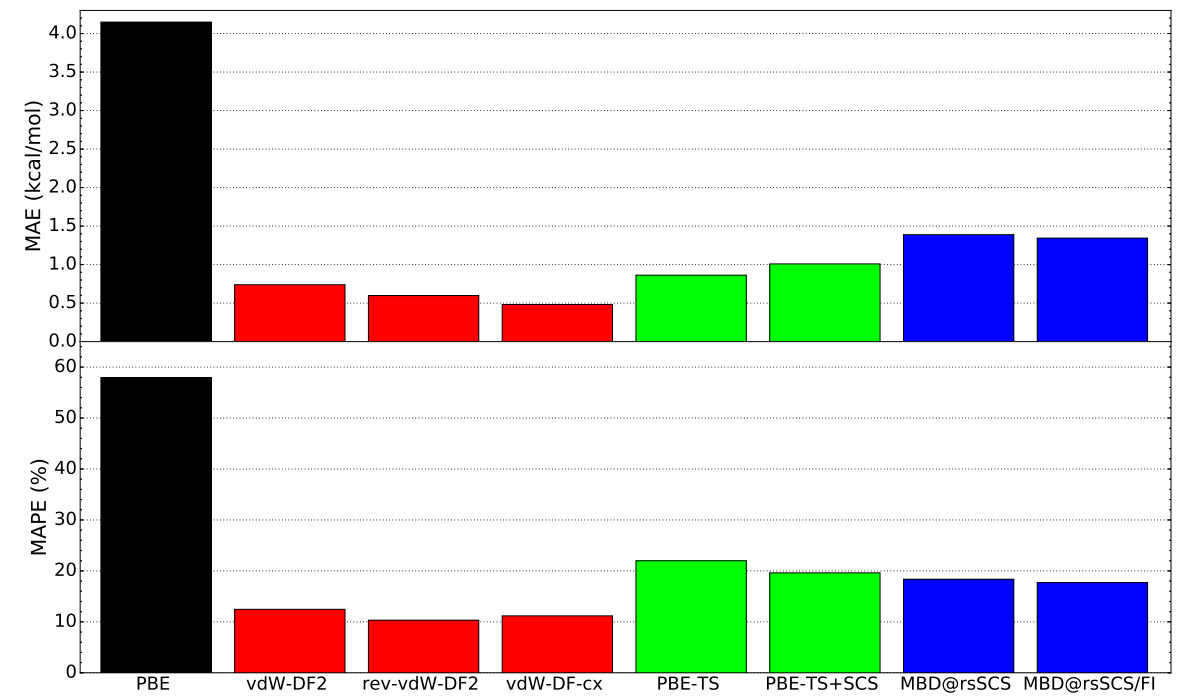

FIG. 4: Mean Absolute Error (MAE) of interaction energies in $\mathrm{kcal} / \mathrm{mol}$ and Mean Absolute Percentage Error (MAPE) in \% for the whole set of molecules (41 molecules) composing the three test sets. Here, all methods show improvements to energies over PBE, with the vdW-DF methods outperforming TS and MBD methods.

structures, despite giving good energies ${ }^{65-67}$. Further testing should be carried out in this regard to understand which approaches can consistently reproduce structures and forces.

Before concluding, let us take a brief moment to consider one of the more surprising findings from this work, that the MBD and FI methods perform more poorly than straight TS theory or any of the vdW-DF type approximations ${ }^{68}$. This holds true (at least for absolute errors, for percentage errors the situation is reversed) even in the larger systems (PHE and GGG complexes from the L7 test set) where many-body effects (specifically many-atom effects in these theories) might be expected to perform better due to greater numbers of 3-, 4- and $n$-atom terms. This combinatorial picture, while compelling, misses an important aspect of such terms, however - that the next-dominant (in gapped insulators) 3-body terms introduce repulsive and attractive contributions [equivalent to plus and minus signs on $x_{n}$ in Eq. (16)], and can thus cancel each other out. A more physical perspective is that most field lines crowd each other out, leaving only the direct contributions between pairs of atoms.

There is thus no reason to assume the many-body terms will necessarily contribute systematically to the energy as a function of size. Indeed dispersion between two bulk insulators 
or a bulk insulator and an atom or small molecule can be quite well represented by sums over their constituent atoms provided the underlying polarizability model is sufficiently good, which enables the broad success of Lifshitz theory ${ }^{69}$. The exception to this argument is in systems like 2D materials and nanowires where the geometry is strongly anisotropic and thus favours one sign over another, as identified by Dobson and co-workers ${ }^{70-73}$. None of the systems studied in this work fall into this category, however. Thus, contributions from many-body terms are unlikely to help or hinder in any systematic way the treatment of dispersion for the systems reported here.

Finally, several directions can be investigated for possible improvements to the methods used here. Firstly, the performance of the TS family of methods depends on the exchangecorrelation functional with which it is combined. It was demonstrated previously ${ }^{74}$ that combining the TS and MBD methods with a different exchange-correlation functional can lead to improvements. Here PBE was used, as the presence of an exact exchange contribution increases significantly the computational cost in plane wave codes and would therefore make molecular dynamics calculations difficult for relatively large systems. Secondly, the vdwDF functionals are based on a simplified version of the adiabatic connection fluctuation dissipation theorem ${ }^{75}$, to which several simplifications have been introduced. Having a new $\operatorname{look}^{76}$ at each of them to work out where precision can be gained would be helpful to improve existing methods. Thirdly, improvements can be made to the MBD models, through further refinement of the polarizability model (as per Ref. 34) and inclusion of Type-C contributions (from Dobson's classifications of non-additivity ${ }^{35}$ ) for large metallic systems. Nonetheless, the current state-of-art does a good job for the systems considered here and, should perform well for similar systems.

\section{ACKNOWLEDGMENTS}

The authors acknowledge financial support from the Agence Nationale de la Recherche under Grant No. ANR-15-CE29-0003-01, from the CNRS PICS program 2DvdW, from the French-Korean STAR program (NRF-2017K1A3A1A21013734), and from the French PIA project "Lorraine Université d'Excellence". Calculations were conducted using GENCICCRT/CINES computational resources under Grant No. x2017-085106 and the DECI resource ARCHER based in the United Kingdom within PRACE aisbl. The authors acknowl- 
edge Torbjörn Björkman for making his implementation of the vdW-DF-cx functional in the VASP code available to them.

\section{REFERENCES}

${ }^{1}$ P. Hohenberg and W. Kohn, Phys. Rev. 136, B864 (1964).

${ }^{2}$ W. Kohn and L. J. Sham, Phys. Rev. 140, A1133 (1965).

${ }^{3}$ S. Grimme, J. Comp. Chem. 25, 1463 (2004).

${ }^{4}$ S. Grimme, J. Comp. Chem. 27, 1787 (2006).

${ }^{5}$ S. Grimme, J. Antony, S. Ehrlich, and H. Krieg, J. Chem. Phys. 132, 154104 (2010).

${ }^{6}$ A. Tkatchenko and M. Scheffler, Phys. Rev. Lett. 102, 073005 (2009).

${ }^{7}$ A. Tkatchenko, R. A. Di Stasio, R. Car, and M. Scheffler, Phys. Rev. Lett. 108, 236402 (2012).

${ }^{8}$ W. J. Kim, M. Kim, E. K. Lee, S. Lebègue, and H. Kim, J. Phys. Chem. Lett. 7, 3278 (2016).

${ }^{9}$ M. Kim, W. J. Kim, E. K. Lee, S. Lebègue, and H. Kim, Int. J. Quantum Chem. 116, $598(2016)$.

${ }^{10}$ M. Dion, H. Rydberg, E. Schroder, D. C. Langreth, and B. I. Lundqvist, Phys. Rev. Lett. 92, 246401 (2004).

${ }^{11}$ O. A. Vydrov and T. R. Voorhis, J. Chem. Phys. 133, 244103 (2010).

${ }^{12}$ K. Berland and P. Hyldgaard, Phys. Rev. B 89, 035412 (2014).

${ }^{13}$ D. C. Langreth and J. P. Perdew, Phys. Rev. B 15, 2884 (1977).

${ }^{14}$ D. Langreth and J. Perdew, Solid State Commun. 17, 1425 (1975).

${ }^{15}$ F. Furche, Phys. Rev. B 64, 195120 (2001).

${ }^{16}$ A. Dixit, J. G. Ángyán, and D. Rocca, J. Chem. Phys. 145, 104105 (2016).

${ }^{17}$ A. Dixit, J. Claudot, S. Lebègue, and D. Rocca, J. Chem. Theory Comput. 13, 5432 (2017).

${ }^{18}$ C. Møller and M. S. Plesset, Phys. Rev. 46, 618 (1934).

${ }^{19}$ R. J. Bartlett and M. Musiał, Rev. Mod. Phys. 79, 291 (2007).

${ }^{20}$ B. Jeziorski, R. Moszynski, and K. Szalewicz, Chem. Rev. 94, 1887 (1994).

${ }^{21}$ A. J. Misquitta, B. Jeziorski, and K. Szalewicz, Phys. Rev. Lett. 91, 033201 (2003).

${ }^{22}$ A. Heßelmann and G. Jansen, Chem. Phys. Lett. 367, 778 (2003). 
${ }^{23}$ O. Gunnarsson and B. I. Lundqvist, Phys. Rev. B 13, 4274 (1976).

${ }^{24}$ W. A. Al-Saidi, V. K. Voora, and K. D. Jordan, J. Chem. Theory Comput. 8, 1503 (2012).

${ }^{25}$ J. Kilmeš, D. Bowler, and A. Michaelides, J. Phys.: Cond. Matt. 22, 022201 (2010).

${ }^{26}$ A. Otero-de-la Roza and E. R. Johnson, J. Chem. Phys. 137, 054103 (2012).

${ }^{27}$ J. Hermann, R. A. DiStasio, and A. Tkatchenko, Chem. Rev. 117, 4714 (2017).

${ }^{28}$ K. Berland, V. R. Cooper, K. Lee, E. Schroder, T. Thonhauser, P. Hyldgaard, and B. I. Lundqvist, Reg. Prog. Phys. 78, 066501 (2015).

${ }^{29}$ J. Řezáč and P. Hobza, J. Chem. Theory Comput. 9, 2151 (2013).

${ }^{30}$ R. Sedlak, T. Janowski, M. Pitoňák, J. Řezáč, P. Pulay, and P. Hobza, J. Chem. Theory Comput. 9, 3364 (2013).

${ }^{31}$ D. E. Taylor, J. G. Ángyán, G. Galli, C. Zhang, F. Gygi, K. Hirao, J. W. Song, K. Rahul, O. Anatole Von Lilienfeld, R. Podeswa, I. W. Bulik, T. M. Henderson, G. E. Scuseria, J. Toulouse, R. Peverati, D. G. Truhlar, and K. Szalewicz, J. Chem. Phys. 145, 124105 (2016).

${ }^{32}$ A. Tkatchenko, A. Ambrosetti, and R. A. DiStasio, Jr., J. Chem. Phys. 138, 074106 (2013).

${ }^{33}$ A. Ambrosetti, A. M. Reilly, R. A. DiStasio Jr, and T. Tkatchenko, J. Chem. Phys. 140, 18A508 (2014).

${ }^{34}$ T. Gould, S. Lebègue, J. G. Ángyán, and T. Bučko, J. Chem. Theory Comput. 12, 5920 (2016).

${ }^{35}$ J. F. Dobson, Int. J. Quantum Chem. 114, 1157 (2014).

${ }^{36}$ J. R. Reimers, M. Li, D. Wan, T. Gould, and M. J. Ford (Elsevier, 2017) pp. 387-416.

${ }^{37}$ J. P. Perdew, K. Burke, and M. Ernzerhof, Phys. Rev. Lett. 77, 3865 (1996).

${ }^{38}$ H. Rydberg, M. Dion, N. Jacobson, E. Schroder, P. Hyldgaard, S. I. Simak, D. C. Langreth, and B. I. Lundqvist, Phys. Rev. Lett. 91, 126402 (2003).

${ }^{39}$ A. Puzder, M. Dion, and D. C. Langreth, J. Chem. Phys 124, 164105 (2006).

${ }^{40}$ K. Lee, E. D. Murray, L. Kong, B. I. Lundqvist, and D. C. Langreth, Phys. Rev. B 82, 081101 (2010).

${ }^{41}$ J. P. Perdew and Y. Wang, Phys. Rev. B 33, 8800(R) (1986).

${ }^{42}$ I. Hamada, Phys. Rev. B 89, 121103 (2014).

${ }^{43}$ A. D. Becke, J. Chem. Phys 85, 7184 (1986).

${ }^{44}$ D. C. Langreth and S. Vosko, Adv. Quantum. Chem. 21, 175 (1990). 
${ }^{45}$ K. Berland, C. A. Arter, V. R. Cooper, K. Lee, B. I. Lundqvist, E. Schroder, T. Thonhauser, and P. Hyldgaard, J. Chem. Phys. 140, 18A539 (2014).

${ }^{46}$ A. D. Becke and E. R. Johnson, J. Chem. Phys. 127, 154108 (2007).

${ }^{47}$ E. R. Johnson and A. D. Becke, J. Chem. Phys. 124, 174104 (2006).

${ }^{48}$ S. Grimme, S. Ehrlich, and L. Goerigk, J. Comput. Chem. 32, 1456 (2011).

${ }^{49}$ N. Marom, A. Tkatchenko, M. Scheffler, and L. Kronik, J. Chem. Theory Comput. 6, 81 (2010).

${ }^{50}$ H. B. G. Casimir and D. Polder, Phys. Rev. 73, 360 (1948).

${ }^{51}$ R. A. DiStasio Jr, O. A. von Lilienfeld, and T. Tkatchenko, Proc. Natl. Acad. Sci. USA 109, $14791(2012)$.

${ }^{52}$ T. Gould and T. Bučko, J. Chem. Theory Comput. 12, 3603 (2016).

${ }^{53}$ Note, in the original manuscript the square bracket was misplaced. The expression used here is correct.

${ }^{54}$ G. Kresse and J. Hafner, Phys. Rev. B 48, 13115 (1993).

${ }^{55}$ G. Kresse and J. Furthmüller, Phys. Rev. B 54, 11169 (1996).

${ }^{56}$ J. Klimeš, D. R. Bowler, and A. Michaelides, Phys. Rev. B 83, 195131 (2011).

${ }^{57}$ G. Román-Pérez and J. M. Soler, Phys. Rev. Lett 103, 096102 (2009).

${ }^{58}$ T. Björkman, J. Chem. Phys 141, 074708 (2014).

${ }^{59}$ T. Bucko, S. Lebègue, J. Hafner, and J. G. Ángyán, Phys. Rev. B 87, 064110 (2013).

${ }^{60}$ T. Bučko, S. Lebègue, T. Gould, and J. G. Ángyán, J. Phys.: Condens. Matter 28, 045201 (2016).

${ }^{61}$ P. E. Blöchl, Phys. Rev. B 50, 17953 (1994).

${ }^{62}$ G. . Kresse and J. Joubert, Phys. Rev. B. 59, 1758 (1999).

${ }^{63}$ F. Gygi, IBM J. Res. \& Dev. 52 (2008).

${ }^{64}$ P. Jurečka, J. Cerný, P. Hobza, and D. R. Salahub, J. Comput. Chem. 28, 555 (2007).

${ }^{65}$ S. Lebègue, J. Harl, T. Gould, J. G. Ángyán, G. Kresse, and J. F. Dobson, Phys. Rev. Lett. 105, 196401 (2010).

${ }^{66}$ T. Björkman, A. Gulans, A. V. Krasheninnikov, and R. M. Nieminen, Phys. Rev. Lett. 108, 235502 (2012).

${ }^{67}$ S. A. Tawfik, T. Gould, C. Stampfl, and M. J. Ford, arXiv preprint arXiv:1712.08327 (2017).

${ }^{68}$ S. Grimme, A. Hansen, J. G. Brandenburg, and C. Bannwarth, Chem. Rev. 116, 5105 
(2016).

${ }^{69}$ E. M. Lifshitz, J. Exper. Theoret. Phys. USSR 29, 94 (1955).

${ }^{70}$ T. Bučko, J. Hafner, S. Lebègue, and J. G. Ángyán, J. Phys. Chem. A 114, 11814 (2010).

${ }^{71}$ J. F. Dobson and T. Gould, J. Phys.: Condens. Matter 24, 073201 (2012).

${ }^{72}$ J. F. Dobson, T. Gould, and G. Vignale, Phys. Rev. X 4, 021040 (2014).

${ }^{73}$ J. F. Dobson, Internation Journal of Quantum Chemistry 114, 1157 (2014).

${ }^{74}$ N. Marom, A. Tkatchenko, M. Rossi, V. V. Gobre, O. Hod, M. Scheffler, and L. Kronik, J. Chem. Theory Comput. 7, 3944 (2011).

${ }^{75}$ M. Fuchs and X. Gonze, Phys. Rev. B 65, 235109 (2002).

${ }^{76}$ J. F. Dobson and T. Gould, J. Phys.: Condens. Matter 24, 073201 (2012). 